\title{
Three-week schedule of irinotecan plus cisplatin in patients with previously untreated extensive-stage small-cell lung cancer
}

\author{
YS Hong',2, HR Lee', ${ }^{1,2}$ S Park', SC Lee', IG Hwang', B-B Park', J Lee', JS Ahn', M-J Ahn', HY Lim' and \\ K Park*,i \\ 'Division of Hematology-Oncology, Department of Medicine, Samsung Medical Center, Sungkyunkwan University School of Medicine, Seoul, Korea
}

Irinotecan and cisplatin demonstrated promising outcomes in extensive-stage small-cell lung cancer. According to the dosage and schedule of irinotecan, efficacy and toxicity profiles showed subtle differences. This study was designed to evaluate efficacy and toxicity of 3-week schedule of irinotecan/cisplatin in patients with previously untreated extensive-stage small-cell lung cancer. The primary objective was to evaluate response rate and secondary objectives were overall survival and progression-free survival. Patients with previously untreated extensive-stage small-cell lung cancer were enrolled. Irinotecan $65 \mathrm{mg} \mathrm{m}^{-2}$ was administered on days I and 8 and cisplatin $60 \mathrm{mg} \mathrm{m}^{-2}$ on day I. Treatment was repeated every 3 weeks. Seven out of 54 patients (I3.0\%) had complete response, and partial response was observed in 33 (61.1\%). The overall response rate was 74.1\% (95\% Cl; 62.0-82.2\%). Stable disease was observed in eight (14.8\%) and no progressive disease was observed. After a median follow-up duration of 28.7 months, the median overall survival and progressive-free survival were 13.6 and 6.5 months, respectively. Major grade 3/4 toxicities were neutropenia (50.0\%), anorexia (42.6\%), diarrhoea (29.6\%), fatigue (29.6\%) and vomiting (13.0\%). There was one treatment-related death owing to pneumonia. Three-week schedule of irinotecan/cisplatin showed effective antitumour activity and moderate toxicities in patients with previously untreated extensive-stage small-cell lung cancer.

British Journal of Cancer (2006) 95, 1648- 1652. doi:I0.1038/sj.bjc.6603500 www.bjcancer.com

Published online 28 November 2006

(c) 2006 Cancer Research UK

Keywords: three-week schedule; irinotecan; cisplatin; extensive-stage small-cell lung cancer

Lung cancer is the leading cause of cancer-related deaths in Western countries (Jemal et al, 2004), and also in Korea (Shin et al, 2004). The proportion of histologic diagnosis with small-cell lung cancer (SCLC) in the United States is approximately $15 \%$ of patients with lung cancer (Jemal et al, 2004), and more than half of these patients are diagnosed with extensive-stage disease. Incidence of SCLC had paralleled trends in cigarette smoking, and smoking prevalence for the adult population is relatively high in Korea. During the last decade, most patients with extensivestage SCLC (ES-SCLC) have been treated with etoposide and platinum resulting in a median survival of 8-10 months (Roth et al, 1992). Although several trials of platinum-based combination chemotherapy were performed, it failed to show a superiority to combination of etoposide and cisplatin (EP) (Mavroudis et al, 2001; Sundstrom et al, 2002).

Irinotecan, which inhibits the function of topoisomerase I in cancer cells, demonstrated synergism and non-cross resistance when combined with platinum agents (Fukuda et al, 1996; Masuda et al, 1996). Kudoh et al (1998) reported that combination chemotherapy of irinotecan and cisplatin (IP) in patients with

\footnotetext{
*Correspondence: Dr K Park, Division of Hematology/Oncology, Department of Medicine, Samsung Medical center, Sungkyunkwan University School of Medicine, 50 Irwon-dong Gangnam-gu, Seoul 135 7I0 Korea; E-mail: kpark@smc.samsung.co.kr

2 These authors contributed equally to this work.

Received 10 August 2006; revised 30 October 2006; accepted 30 October 2006; published online 28 November 2006
}

SCLC showed a promising response rate of 84 with $29 \%$ of complete responses and median survival over 13 months. Since then, several phase II/III trials of irinotecan-based combination chemotherapy with different dose and schedule were performed with varying results in SCLC patients (Noda et al, 2002; Kudoh et al, 2005; Hanna et al, 2006; Kinoshita et al, 2006; Schmittel et al, 2006). In a previous phase III trial conducted by a Japanese group, patients were treated with $60 \mathrm{mg} \mathrm{m}^{-2}$ of cisplatin on day 1 and $60 \mathrm{mg} \mathrm{m}^{-2}$ of irinotecan on days 1,8 , and 15 every 4 weeks, and showed a good median survival of 12.8 months. However, only $80.4 \%$ of planned dose of irinotecan was administered and the day15 irinotecan was omitted in $50 \%$ of patients owing to toxicities (Noda et al, 2002). Another phase III trial performed in the West tested $65 \mathrm{mg} \mathrm{m}^{-2}$ of irinotecan on days 1 and 8 every 3 weeks in ES-SCLC patients, and showed rather lower median survival of 9.3 months (Hanna et al, 2006) compared with the Japanese trial, suggesting possible ethnic and pharmacogenomic differences between the two study populations.

Based on these results, we conducted a phase II study using irinotecan $65 \mathrm{mg} \mathrm{m}^{-2}$ on days 1 and 8 , and cisplatin $60 \mathrm{mg} \mathrm{m}^{-2}$ on day 1 schedule in chemotherapy-naïve ES-SCLC patients to evaluate the efficacy and safety of the regimen in Korean patients.

\section{PATIENTS AND METHODS}

\section{Patients' eligibility}

Patients with previously untreated ES-SCLC were enrolled. Patients were eligible if they had (1) histologically confirmed small-cell 
carcinoma; (2) extensive-stage disease; (3) Eastern Cooperative Oncology Group (ECOG) performance status (PS) of $\leqslant 3$; (4) age over 18 years old; (5) adequate haematologic parameters (haemoglobin $\geqslant 9.0 \mathrm{~g} \mathrm{dl}^{-1}$, absolute neutrophil count $\geqslant 1500 \mu \mathrm{l}^{-1}$, platelet count $\geqslant 100000 \mu \mathrm{l}^{-1}$ ), renal functions (serum creatinine $\leqslant 1.5 \mathrm{mg} \mathrm{dl}^{-1}$ or calculated creatinine clearance by Cockroft formula $\geqslant 50 \mathrm{ml} \mathrm{min}^{-1}$ ), and hepatic function (aspartate aminotransferase, alanine aminotransferase $\leqslant 3 \times$ upper limits of normal, total bilirubin $\leqslant 2 \times$ upper limits of normal); (6) at least one bi-dimensionally measurable lesion according to the WHO criteria (Miller et al, 1981); (7) absence of active infection; (8) no prior chemotherapy, radiotherapy, or surgery for the disease; (9) no history of myocardial infarction in the last 6 months before the study entry; (10) no uncontrolled congestive heart failure or significant arrhythmia; and (11) no prior second primary cancer, except for cervix cancer in situ or skin cancer. Patients with brain metastases were allowed provided that there were no significant neurologic symptoms or signs. All patients provided written informed consent before they entered the study. This protocol was approved by the institutional review board at Samsung Medical Center.

\section{Treatment and dose modification}

Patients were treated with irinotecan $65 \mathrm{mg} \mathrm{m}^{-2}$ on days 1 and 8 , and cisplatin $60 \mathrm{mg} \mathrm{m}^{-2}$ on day 1 . Treatment cycles were repeated every 3 weeks until the maximum six cycles initially planned, documented disease progression, unacceptable toxicity, or patient's refusal. Cisplatin was given with adequate hydration of 21 of intravenous fluid to protect renal functions and with diuretics if needed to control volume status. Cholinergic symptoms that occurred during or within $1 \mathrm{~h}$ after irinotecan administration were treated with atropine $(0.3 \mathrm{mg}$ or as needed). Loperamide was provided as therapy for prophylaxis of delayed diarrhoea. A 5hydroxytryptamine type- 3 receptor antagonist was given as emesis prophylaxis before drug administration.

Application of chemotherapy was delayed by one or two weeks until haematologic recovery of absolute neutrophil count $\geqslant 1500 \mu \mathrm{l}^{-1}$ and platelet $\geqslant 100000 \mu \mathrm{l}^{-1}$. Administration of irinotecan was omitted on day eight if grade 2 or 3 diarrhoea occurred, and subsequent cycles were allowed when the diarrhoea recovered to baseline or grade $\leqslant 1$. The dose of irinotecan in subsequent cycles was reduced by $25 \%$ from the planned dose if there were any grade 4 hematologic toxicities lasting more than 7 days, $\geqslant$ grade 3 haematologic toxicities with febrile episode, or bleeding-associated thrombocytopenia. Treatment was discontinued in patients with grade 4 diarrhoea. The dose of cisplatin in subsequent cycles was reduced by $25 \%$ from the planned dose if there were grade 4 haematologic toxicities or if grade 2 renal toxicity. In the presence of grade 3 or 4 non-haematologic toxicity (except nausea, vomiting, and alopecia), the treatment was postponed until resolution of the toxicity and then both drug doses were reduced by $25 \%$ for the next cycle. Once a dose reduction was required, re-escalation of dose was not allowed.

\section{Assessment of efficacy and toxicity}

The following pretreatment evaluations were performed within 2 weeks before study entry: a full medical history and physical examination, complete blood cell count with differentials, chemistry profiles, urinalysis, and performance status evaluation. Chest $\mathrm{X}$-rays, chest and upper abdominal computed tomography (CT) scans, brain magnetic resonance imaging, radionuclide bone scan and any other diagnostic procedures as clinically indicated were performed within 4 weeks before enrollment. During treatment, a limited history taking, physical examination, assessment of toxicity, complete blood cell count with differentials, blood chemistry and chest X-rays were performed every 3 weeks before each cycle. Appropriate imaging studies including CT scans of chest and upper abdomen were performed every two cycles (6 weeks) to assess treatment response, and sooner if needed for documentation of disease progression. Objective tumour responses were assessed according to the WHO criteria (Miller et al, 1981). Complete response (CR) was defined as the disappearance of all known disease for at least 4 weeks with no new lesion appearing. Partial response (PR) referred to an at least $50 \%$ decrease in the sum of the products of bidimensional diametres lasting for at least 4 weeks without the appearance of new lesions. Stable disease (SD) was defined as failure to observe a PR or CR, no progressive or new lesions were observed for at least 4 weeks. Progressive disease (PD) was defined as a $25 \%$ or greater increase in the sum of the products of bidimensional diameters of any measurable lesion or the appearance of new lesions. All enrolled patients were included in the intention-to-treat analysis of efficacy. Response rate was calculated as the ratio of the number of patients who achieved complete or partial responses to the number of enrolled patients. Overall survival (OS) and progression-free survival (PFS) were calculated from the start of therapy until death and progression, respectively, or until last follow-up. Toxicities were monitored according to the National Cancer Institute Common Toxicity Criteria (NCI-CTC) scale version 3.0.

\section{Statistical consideration}

The sample size was calculated according to Simon's two-stage optimal design (Simon, 1989). A targeted objective response rate of $80 \%$ versus an objective response rate of no interest of $60 \%$ with a power of 0.90 at a one-sided significance level of 0.05 was chosen, and accrual of 45 assessable patients was projected. In the initial stage, 18 evaluable patients were to be entered into the study and evaluated for response. If there were $>11$ responses, accrual was to be terminated. If $\geqslant 11$ responses were observed in the first stage, then 27 additional patients were to be entered in the second stage to achieve a target sample size of 45 evaluable patients. Assuming that $10 \%$ of patients were inassessable, at least a total of 50 patients were planned to be accrued for this study. All patients who received at least one course of therapy were considered assessable for toxicity, and all eligible patients who received at least one cycle of therapy were included for survival estimation. Descriptive statistics were reported as proportions and medians. OS and PFS were assessed by the Kaplan-Meier method and the $95 \%$ confidence interval (95\% CI) for the median time to event was computed.

\section{RESULTS}

\section{Patient characteristics}

Between November 2002 and January 2005, 54 patients were enrolled and their clinical characteristics are shown in Table 1 . The median age was 64 years (range, $47-78$ years), 49 patients $(90.7 \%)$ were male and five $(9.3 \%)$ were female. Forty patients $(74.1 \%)$ had ECOG PS of $0-1$, whereas 14 patients (25.9\%) had ECOG PS of 2 or 3 . The most common sites of metastasis were lymph nodes $(40 / 54$, $74.1 \%)$ and brain metastasis was observed in 17 patients $(31.5 \%)$ at the time of enrollment. Median serum lactate dehydrogenase (LDH) level at initial diagnosis was $408 \mathrm{IUl}^{-1}$ (range, 225-1566).

\section{Drug administration}

Drug administration and relative dose intensity are shown in Table 2. The median number of cycles to be administered was four (range 1-6 cycles), and 24 (44.4\%) patients completed the planned six cycles of chemotherapy. Treatment was delayed for a median of 2 weeks in 109 out of subsequent 223 cycles (48.9\%). The most common cause for delayed administration was neutropenia (58 
Table I Patient characteristics

\begin{tabular}{|c|c|c|}
\hline Characteristics & $\begin{array}{c}\text { No. of patients } \\
54\end{array}$ & $\begin{array}{c}\% \\
100\end{array}$ \\
\hline Age, years (range) & Median $64(47-78)$ & \\
\hline \multicolumn{3}{|l|}{ Sex } \\
\hline Male & 49 & 90.7 \\
\hline Female & 5 & 9.3 \\
\hline \multicolumn{3}{|l|}{ ECOG PS } \\
\hline 0 & 2 & 3.7 \\
\hline । & 38 & 70.4 \\
\hline 2 & 10 & 18.5 \\
\hline 3 & 4 & 7.4 \\
\hline \multicolumn{3}{|l|}{ No. of distant metastatic sites } \\
\hline 0 & 9 & 16.7 \\
\hline I & 20 & 37.0 \\
\hline 2 & 14 & 25.9 \\
\hline$\geqslant 3$ & 11 & 20.4 \\
\hline \multicolumn{3}{|l|}{ Metastatic sites } \\
\hline Lymph nodes & 40 & 74.1 \\
\hline Brain & 17 & 31.5 \\
\hline Bone & 16 & 29.6 \\
\hline Pleural effusion or seeding & 16 & 29.6 \\
\hline Liver & 14 & 25.9 \\
\hline Adrenal glands & 11 & 20.4 \\
\hline Initial LDH (IUI) levels & Median $408(225-1566)$ & \\
\hline
\end{tabular}

$E C O G=$ Eastern Cooperative Oncology Group; $L D H=$ lactate dehydrogenase; $\mathrm{PS}=$ performance status.

Table 2 No. of chemotherapy cycles and delivered actual dose

\begin{tabular}{lcc}
\hline Cycles & $\begin{array}{c}\text { No. of patient } \\
\text { (\%) }\end{array}$ \\
\hline 1 & $7(13.0)$ \\
2 & $9(16.7)$ & \\
3 & $4(7.4)$ & \\
4 & $8(14.8)$ & \\
5 & $2(3.7)$ & \\
6 & $24(44.4)$ & 87.0 \\
& Delivered dosel \\
planned dose & $\mathbf{~ m g ~ m}^{-2} \mathbf{w e e k}^{-\mathbf{1}} \mathbf{)}$ \\
\% of planned dose & $17.4 / 20.0$ & 84.5 \\
Cisplatin & $36.6 / 43.3$ & \\
Irinotecan & & \\
\hline
\end{tabular}

cycles, 53.3\%). Twenty-two cycles (9.9\%) required dose reductions mainly due to neutropenia and diarrhoea. The delivered dose intensity (DI) was $17.4 \mathrm{mg} \mathrm{m}^{-2}$ week $^{-1}$ ( $87.0 \%$ of planned dose) for cisplatin and $36.6 \mathrm{mg} \mathrm{m}^{-2}$ week $^{-1}$ (84.5\% of planned dose) for irinotecan.

\section{Response}

Forty-eight (88.9\%) of 54 patients were evaluable for responses. Six patients were not evaluable, but were included in the intent-totreat analysis. Two patients refused to receive chemotherapy after first cycle of the regimen and were lost to follow-up, one patient was referred to another hospital, one patient was dropped out after first dose of the regimen owing to poor performance status, one patient underwent operation for gastric ulcer perforation, and there was one treatment-related death.

The overall response rate was $74.1 \%$ (95\% CI; $62.0-82.2 \%$ ), with a complete response rate of $13 \%$ (Table 3). SD was observed in eight patients $(14.8 \%)$ on initial assessment of response.

\section{Survival}

All 54 patients were included in the survival analysis on an intentto-treat basis. The OS and PFS of patients in this study is shown in Figure 1. After the median follow-up duration of 28.7 months (range, 10.9-45.0 months), the median overall survival was 13.6 months (95\% CI, 10.7-15.5 months) and 1 year survival rate was $53.1 \%$. The median PFS was 6.5 months (95\% CI, 5.1 - 7.9 months) and 1 year PFS was $10.5 \%$.

\section{Toxicities}

All patients were evaluable for toxicities, and grade 3 or 4 toxicities observed during treatment are listed in Table 4. Haematologic toxicity was most prevalent in this study. Grade 3 or 4 neutropenia occurred in $27(50.0 \%)$ of 54 patients. Grade 3 or 4 anaemia and

Table 3 Objective responses

\begin{tabular}{lcc}
\hline Tumour response $(\boldsymbol{n}=\mathbf{5 4})$ & No. of patients & $\%$ \\
\hline Complete response* & 7 & 13.0 \\
Partial response* & 33 & 61.1 \\
Stable disease & 8 & 14.8 \\
Progressive disease & 0 & 0 \\
Not evaluable & 6 & 11.1 \\
\hline
\end{tabular}

*Overall response rate: $74.1 \%$ (95\% Cl, 62.0-86.2).
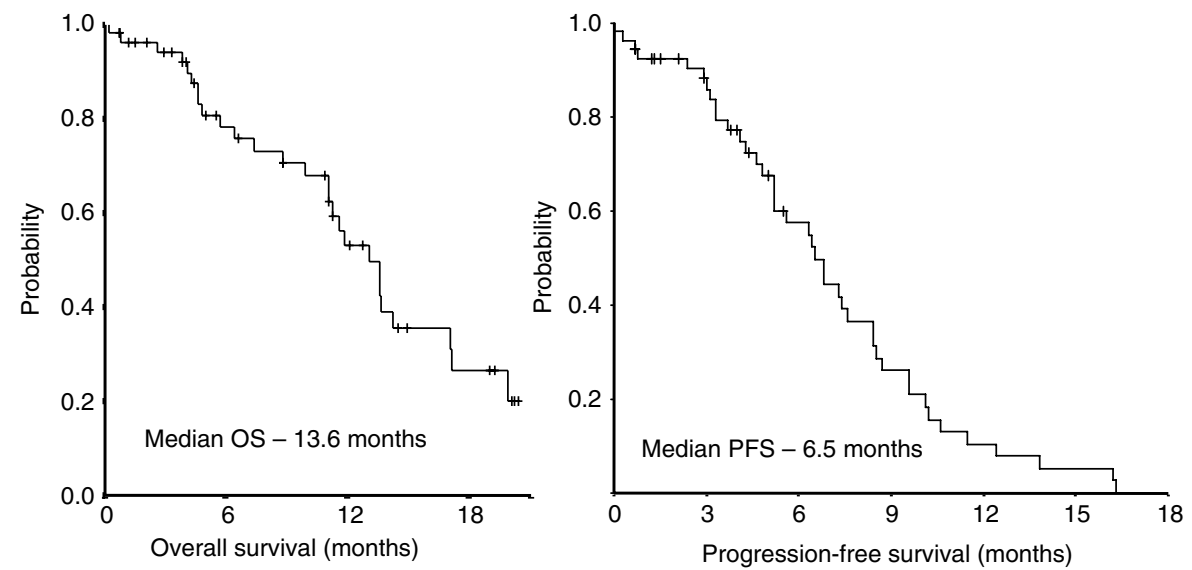

Figure I OS and PFS of the all patients. 
thrombocytopenia were observed in two $(3.7 \%)$ of 54 patients, respectively. Most significant $\geqslant$ grade 3 non-haematologic toxicity was anorexia $(n=23,42.6 \%)$. Grade 3 or 4 diarrhoea occurred in $16(29.6 \%)$ patients, with grade 2 diarrhoea in seven $(13.0 \%)$ patients and grade 1 diarrhoea in $22(40.7 \%)$ patients. In addition, $24(44.4 \%)$ patients showed grade 2 alopecia and seven $(13.0 \%)$ showed grade 2 neuropathy. There was one treatment-related death. That patient was a 51-year-old male with ECOG performance status score of 3 , and had brain metastasis at initial presentation of disease. He developed neutropenic sepsis with pneumonia caused by Streptococcus pneumoniae on day 19 and died of aggravation of pneumonia on day 26 of the first cycle of chemotherapy despite recovery from neutropenia.

\section{Salvage chemotherapy}

Of 55 patients, 24 patients $(43.6 \%)$ were treated with systemic chemotherapy as second-line treatment, and 22 patients were evaluable for treatment responses. Salvage regimens included etoposide and carboplatin $(11 / 24,45.8 \%)$, EP (10/24, $41.7 \%)$, oral etoposide and carboplatin (2/24, 8.3\%), and paclitaxel and ifosfamide $(1 / 24,4.2 \%)$. By intent-to-treat analysis, overall response rate of salvage treatment was 37.5\% (95\% CI, 18.1 $56.9 \%$ ) with one CR and eight PR. SD and PD were observed in four patients (16.7\%) and nine patients (37.5\%), respectively. Median time to progression (TTP) was 3.4 months (1.0-20.0 months) in all 24 patients and median duration of response was 3.6 months (1.6-19.8 months) in responders. We defined chemosensitive relapse as PD was noted after 3 months of maximal response, and primary refractory disease as initial maximal response was $\mathrm{SD}$, or $\mathrm{PD}$ was noted within 3 months of maximal response. As our definition, 17 patients $(17 / 24,70 \%)$ showed chemo-sensitive relapse, and seven patients (7/24, 30\%) showed primary refractory disease. In 17 patients with chemo-sensitive relapse, responses to second-line chemotherapy were observed in five patients (29.4\%, with one CR and four PR.), SD in three patients (17.6\%), and PD in seven patients (41.2\%). Two of 17 patients with chemo-sensitive relapse were inevaluable. In seven patients with primary refractory disease, response to second-line chemotherapy was observed in four patients $(57.1 \%$, with zero CR and four PR), SD in one patient (14.3\%), and PD in two patients $(28.6 \%)$. Response differences in these two groups did not show statistical significance $(P=0.531)$.

\section{DISCUSSION}

For treatment for extensive-stage SCLC, combination chemotherapy with EP has been the standard regimen since the last decade.

Table 4 Toxicity profiles

\begin{tabular}{|c|c|c|c|c|}
\hline & \multicolumn{2}{|l|}{ Grade 3} & \multicolumn{2}{|l|}{ Grade 4} \\
\hline & No. of patients & $\%$ & No. of patients & $\%$ \\
\hline \multicolumn{5}{|l|}{ Hematologic toxicities } \\
\hline Neutropenia & 18 & 33.3 & 9 & 16.7 \\
\hline Thrombocytopenia & 0 & 0.0 & 2 & 3.7 \\
\hline Anaemia & 2 & 3.7 & 0 & 0.0 \\
\hline Febrile neutropenia & 7 & 13.0 & I & 1.9 \\
\hline \multicolumn{5}{|l|}{ Nonhematologic toxicities } \\
\hline Anorexia & 22 & 40.7 & I & 1.9 \\
\hline Diarrhoea & 15 & 27.7 & 1 & 1.9 \\
\hline Fatigue & 15 & 27.7 & 1 & 1.9 \\
\hline Nausea/vomiting & 7 & 13.0 & 0 & 0.0 \\
\hline Stomatitis & 5 & 9.6 & 0 & 0.0 \\
\hline Constipation & 4 & 7.4 & 0 & 0.0 \\
\hline
\end{tabular}

However, combination chemotherapy with IP came into the spotlight after the Japanese phase II trial in 1998 (Kudoh et al, 1998). In 1998, Kudoh et al (1998) reported $84 \%$ of overall response rate with $29 \%$ of complete response and 13 months of median OS in patients with extensive-stage SCLC. In this trial, irinotecan was administrated at $60 \mathrm{mg} \mathrm{m}^{-2}$ on days 1,8 and 15 repeated every 3 weeks. In this dose and schedule of irinotecan, grade 3 or 4 haematologic toxicities were exceedingly frequent with $77 \%$ of neutropenia, $45 \%$ of leucopenia. Despite high incidence of haematologic toxicities, this study was the first to report median survival that exceeds 1 year in ES-SCLC (Kudoh et al, 1998).

The promising preliminary results led to several irinotecanbased phase II and III trials in patients with SCLC. Two most representative phase III trials were published in 2002 and 2006, respectively (Noda et al, 2002; Hanna et al, 2006). In 2002, Noda et al (2002), reported that combination with irinotecan and cisplatin (IP) was superior to that of EP in terms of response rates and OS (65\%, 12.8 months vs 52\%, 9.4 months, $P=0.002)$ for the patients with ES-SCLC. Although IP seemed more effective than $\mathrm{EP}$, the toxicities were higher in the IP arm with $65.3 \%$ of the patients with grade $3 / 4$ neutropenia and $26.7 \%$ with grade $3 / 4$ diarrhoea. In this trial, irinotecan was administered with $60 \mathrm{mg} \mathrm{m}^{-2}$ on days 1,8 and 15 every 4 weeks in combination with $60 \mathrm{mg} \mathrm{m}^{-2}$ of cisplatin on day one, and the relative dose intensity was $80.4 \%$ of the planned dose. Most recently, however, Hanna et al (2006) reported IP did not show survival benefit compared with EP (9.3 vs 10.2 months, $P=0.74)$. This trial adopted $65 \mathrm{mg} \mathrm{m}^{-2}$ of irinotecan on days 1 and 8 every 3 weeks schedule in combination with $30 \mathrm{mg} \mathrm{m}^{-2}$ of cisplatin on day 1 and 8 , and showed lower overall response rate of $48 \%$ and shorter median OS of 9.3 months compared with previous trial of Noda et al (2002), in IP group. The toxicities seemed significantly lower than previous trial with only $36.2 \%$ of patients experiencing grade $3 / 4$ neutropenia in IP group (Hanna et al, 2006).

In this study, irinotecan was administered at $65 \mathrm{mg} \mathrm{m}^{-2}$ on days 1 and 8 , combined with $60 \mathrm{mg} \mathrm{m}^{-2}$ of cisplatin on day 1 in a 3 -week schedule; thus, the dosages would be in the medium range as compared to those used in previous two trials. This regimen showed $74.1 \%$ of response rate and 13.6 months of median OS in the same patient population. It surpasses the results of Kudoh et al (1998) in median OS over 13 months, and showed higher response rates than that of equal dosage of irinotecan (Hanna et al, 2006). These results are very encouraging and superior to other trials with similar irinotecan dosages and schedules. Grade 3/4 toxicities were $50 \%$ in neutropenia and $29.6 \%$ in diarrhoea, and were comparable with previous other trials (Kudoh et al, 1998; Noda et al, 2002; Hanna et al, 2006; Kinoshita et al, 2006). However, one treatmentrelated death was observed in this trial. In the present study, four patients $(7.4 \%)$ had initial poor performance status of ECOG score 3 (Table 1). One patient died during chemotherapy as previously noted, another two patients could not complete the planned treatment owing to toxicities. Only one patient could receive scheduled chemotherapy and showed response of stable disease. As the SCLC is one of the most chemosensitive tumour types, patients with poor performance status $(P S \geqslant 3)$ who are not usually included in clinical trials of other tumour types had often been included in some trials. The present study also included a few patients with poor PS who, however, did not do so well with this treatment as mentioned above. Therefore, special cautions need to be exerted for patients with poor performance status in future trials. Given the considerable toxicities and the median number of cycles to be administered was four cycles, one may also need to consider to shorten the treatment cycles to four from six in future trials.

Irinotecan is metabolised by carboxylesterase to an active metabolite 7-ethyl-10-hydroxycamptothecin (SN-38). $\mathrm{SN}-38$ is then further metabolised in the liver by uridine diphosphateglucuronosyltransferases (UGTs) to an inactive metabolite, SN-38 
glucuronide (SN-38G) (Mathijssen et al, 2001). This glucuronidatioin is the major route of detoxification of irinotecan, thus inherited differences in irinotecan metabolism may have an important influence on the pharmacokinetics and toxicity of this drug. Han et al (2006) reported the UGT1A polymorphisms could predict treatment outcomes and toxicities of irinotecan in Korean patients. In this report, patients with homozygote of UGT1A $1^{\star} 6$ allele had shorter PFS and more irinotecan-related toxicities. Six of 81 Korean patients $(7.4 \%)$ had phenotype of UGT1A ${ }^{*} 6$ homozygosity. Another phenotype associated with low tumour responses and high irinotecan-related toxicity is UGT1A1*28, and it is highly prevalent in Caucasian individuals with reported frequencies of 0.29-0.47 (Bosma et al, 1995; Premawardhena et al, 2003), whereas it has much lower frequency in Asians (0.08$0.19)$ (Yamamoto et al, 1998). In Korean report, the frequency of UGT1A $1 * 28$ was 0.07 (Han et al, 2006). Therefore, it could be explained that ethnic differences might be involved in discrepant results in terms of efficacy and toxicity of irinotecan.

Given the similar schedule and dosage of irinotecan and cisplatin combination chemotherapy in the trial performed by
Hanna et al (2006) compared with our study, higher response rate (74.1 vs 48\%) and longer median survival (13.6 vs 9.3 months) was observed in our study. However, grade 3 or 4 toxicities were more common in our study; neutropenia (50 vs 36.2\%), febrile neutropenia (14.9 vs 3.7\%), and diarrhoea (29.6 vs 21.3\%). Even though the difference in dose and administration schedule, as well as disease characteristics might be plausible explanations of the disparities of outcome and toxicities between the two trials, it might be largely attributable to the pharmacogenomic/ethnic differences as was previously discussed by Hanna et al (2006), Further study with UGT1A polymorphism in this population will be needed.

In conclusion, our results suggest that 3-week schedule of irinotecan $\left(65 \mathrm{mg} \mathrm{m}^{-2}\right.$, on days 1 and 8$)$ and cisplatin $\left(60 \mathrm{mg} \mathrm{m}^{-2}\right.$, on day 1) showed comparable results in terms of response rate, survival, and toxicity with the 4 -week schedule of JCOG. Considering toxicity and convenience, 3-week schedule of irinotecan and cisplatin can be a reasonable option in the treatment of patients with ES-SCLC in Korea, which, however, requires further study in Caucasian population.

\section{REFERENCES}

Bosma PJ, Chowdhury JR, Bakker C, Gantla S, de Boer A, Oostra BA, Lindhout D, Tytgat GN, Jansen PL, Oude Elferink RP, Chowdhury NR (1995) The genetic basis of the reduced expression of bilirubin UDPglucuronosyltransferase 1 in Gilbert's syndrome. $N$ Engl J Med 333: $1171-1175$

Fukuda M, Nishio K, Kanzawa F, Ogasawara H, Ishida T, Arioka H, Bojanowski K, Oka M, Saijo N (1996) Synergism between cisplatin and topoisomerase I inhibitors, NB-506 and SN-38, in human small cell lung cancer cells. Cancer Res 56: 789-793

Han JY, Lim HS, Shin ES, Yoo YK, Park YH, Lee JE, Jang IJ, Lee DH, Lee JS (2006) Comprehensive analysis of UGT1A polymorphisms predictive for pharmacokinetics and treatment outcome in patients with non-small-cell lung cancer treated with irinotecan and cisplatin. J Clin Oncol 24: $2237-2244$

Hanna N, Bunn Jr PA, Langer C, Einhorn L, Guthrie Jr T, Beck T, Ansari R, Ellis P, Byrne M, Morrison M, Hariharan S, Wang B, Sandler A (2006) Randomised phase III trial comparing irinotecan/cisplatin with etoposide/cisplatin in patients with previously untreated extensive-stage disease small-cell lung cancer. J Clin Oncol 24: 2038-2043

Jemal A, Tiwari RC, Murray T, Ghafoor A, Samuels A, Ward E, Feuer EJ, Thun MJ (2004) Cancer statistics, 2004. CA Cancer J Clin 54: 8-29

Kinoshita A, Fukuda M, Soda H, Nagashima S, Fukuda M, Takatani H, Kuba M, Nakamura Y, Tsurutani J, Kohno S, Oka M (2006) Phase II study of irinotecan combined with carboplatin in previously untreated small-cell lung cancer. Br J Cancer 94: 1267-1271

Kudoh S, Fujiwara Y, Takada Y, Yamamoto H, Kinoshita A, Ariyoshi Y, Furuse K, Fukuoka M (1998) Phase II study of irinotecan combined with cisplatin in patients with previously untreated small-cell lung cancer. West Japan lung cancer group. J Clin Oncol 16: 1068-1074

Kudoh S, Nakamura S, Nakano T, Komuta K, Isobe T, Katakami N, Fukuda Y, Takada Y, Takada M, Fukuoka M, Ariyoshi Y (2005) Irinotecan and etoposide for previously untreated extensive-disease small cell lung cancer: a phase II trial of West Japan thoracic oncology group. Lung Cancer 49: $263-269$

Masuda N, Kudoh S, Fukuoka M (1996) Irinotecan (CPT-11): pharmacology and clinical applications. Crit Rev Oncol Hematol 24: 3-26

Mathijssen RH, van Alphen RJ, Verweij J, Loos WJ, Nooter K, Stoter G, Sparreboom A (2001) Clinical pharmacokinetics and metabolism of irinotecan (CPT-11). Clin Cancer Res 7: 2182-2194

Mavroudis D, Papadakis E, Veslemes M, Tsiafaki X, Stavrakakis J, Kouroussis C, Kakolyris S, Bania E, Jordanoglou J, Agelidou M, Vlachonicolis J, Georgoulias V (2001) A multicenter randomised clinical

trial comparing paclitaxel-cisplatin-etoposide versus cisplatin-etoposide as first-line treatment in patients with small-cell lung cancer. Ann Oncol 12: $463-470$

Miller AB, Hoogstraten B, Staquet M, Winkler A (1981) Reporting results of cancer treatment. Cancer 47: $207-214$

Noda K, Nishiwaki Y, Kawahara M, Negoro S, Sugiura T, Yokoyama A, Fukuoka M, Mori K, Watanabe K, Tamura T, Yamamoto S, Saijo N (2002) Irinotecan plus cisplatin compared with etoposide plus cisplatin for extensive small-cell lung cancer. $N$ Engl J Med 346: 85-91

Premawardhena A, Fisher CA, Liu YT, Verma IC, de Silva S, Arambepola M, Clegg JB, Weatherall DJ (2003) The global distribution of length polymorphisms of the promoters of the glucuronosyltransferase 1 gene (UGT1A1): hematologic and evolutionary implications. Blood Cells $\mathrm{Mol}$ Dis 31: $98-101$

Roth BJ, Johnson DH, Einhorn LH, Schacter LP, Cherng NC, Cohen HJ, Crawford J, Randolph JA, Goodlow JL, Broun GO, Omura GA, Greco FA (1992) Randomised study of cyclophosphamide, doxorubicin, and vincristine versus etoposide and cisplatin versus alternation of these two regimens in extensive small-cell lung cancer: a phase III trial of the Southeastern Cancer Study Group. J Clin Oncol 10: $282-291$

Schmittel A, von Weikersthal LF, Sebastian M, Martus P, Schulze K, Hortig P, Reeb M, Thiel E, Keilholz U (2006) A randomised phase II trial of irinotecan plus carboplatin versus etoposide plus carboplatin treatment in patients with extended disease small-cell lung cancer. Ann Oncol 17: $663-667$

Shin HR, J K, Won YJ, Park JG, 139 KCCR-affiliated Hospitals (2004) 2002 annual report of the Korea central cancer registry. Cancer Res Treat 36: $103-114$

Simon R (1989) Optimal two-stage designs for phase II clinical trials. Control Clin Trials 10: $1-10$

Sundstrom S, Bremnes RM, Kaasa S, Aasebo U, Hatlevoll R, Dahle R, Boye N, Wang M, Vigander T, Vilsvik J, Skovlund E, Hannisdal E, Aamdal S (2002) Cisplatin and etoposide regimen is superior to cyclophosphamide, epirubicin, and vincristine regimen in small-cell lung cancer: results from a randomised phase III trial with 5 years' follow-up. J Clin Oncol 20: $4665-4672$

Yamamoto K, Sato H, Fujiyama Y, Doida Y, Bamba T (1998) Contribution of two missense mutations (G71R and Y486D) of the bilirubin UDP glycosyltransferase (UGT1A1) gene to phenotypes of Gilbert's syndrome and Crigler-Najjar syndrome type II. Biochim Biophys Acta 1406: $267-273$ 